\title{
Coagulopathie associée à la COVID-19
}

\author{
Stephanie G. Lee MD MSc, Michael Fralick MD PhD, Michelle Sholzberg MDCM MSc
}

Citation : CMAJ 2020 May 25;192:E583. doi : 10.1503/cmaj.200685-f; diffusion hâtive le $1^{\text {er }}$ mai 2020

Voir la version anglaise de l'article ici : www.cmaj.ca/lookup/doi/10.1503/cmaj.200685

1

Chez $20 \%$ à $55 \%$ des patients hospitalisés pour la maladie à coronavirus 2019 (COVID-19), les analyses de laboratoire montrent des signes de coagulopathie

La coagulopathie est en corrélation avec la gravité de la COVID-19 et pourrait inclure des concentrations élevées de D-dimères ( $\geq 2$ fois la limite supérieure de la normale), un temps de prothrombine légèrement prolongé ( 1-3 s par rapport à la normale), une légère thrombocytopénie (numération plaquettaire $>100 \times 10^{9} / \mathrm{L}$ ) et, plus tard au cours de la maladie, une baisse des taux de fibrinogène $(<2 \mathrm{~g} / \mathrm{L}[5,88 \mu \mathrm{mol} / \mathrm{L}])^{1-3}$. On ignore si la coagulopathie observée est causée directement par le virus ou si elle est secondaire à un état pro-inflammatoire.

\section{Une concentration élevée de D-dimères est associée à un pronostic sombre}

On ignore comment utiliser spécifiquement les taux de D-dimères pour les soins cliniques, mais leur hausse au moment de l'hospitalisation et tout au long du séjour hospitalier est associée à la mortalitét, ${ }^{1,2}$ et un taux de D-dimères 4 fois plus élevé que la normale est associé à un risque environ 5 fois plus grand de maladie grave comparativement à un taux normal (www.medrxiv.org/ content/10.1101/2020.04.08.20057794v1.full.pdf).

La coagulopathie associée à la COVID-19 semble prothrombotique

Dans 2 études rétrospectives, on a diagnostiqué la thromboembolie veineuse chez environ le quart des patients hospitalisés aux soins intensifs pour COVID19,5. Lors d'une étude, la thromboembolie s'est manifestée en dépit de l'administration d'une thromboprophylaxie par héparine de bas poids moléculaire ${ }^{5}$. Les études disponibles n'ont pas mentionné les saignements comme complications fréquentes, mais la littérature scientifique évolue rapidement ${ }^{2,4,5}$.

En l'absence de contre-indications, les patients hospitalisés

devraient recevoir une thromboprophylaxie de routine

La thromboprophylaxie pour prévenir la thromboembolie veineuse est recommandée chez la plupart des patients hospitalisés, surtout chez ceux qui présentent un état pro-inflammatoire. Dans une étude rétrospective, chez les patients qui avaient un taux de $D$-dimères 6 fois plus élevé que la normale et qui ont reçu une thromboprophylaxie par héparine (principalement par énoxaparine à 40-60 mg/j), le taux de mortalité a été moins élevé que chez ceux qui n'ont pas reçu de thromboprophylaxie ${ }^{6}$.

5 La transfusion de produits sanguins est à éviter chez les patients qui ne présentent pas d'hémorragie active La transfusion dans le seul but de corriger les paramètres hémostatiques pourrait être nuisible (p. ex., risque de réaction transfusionnelle), indépendamment du fait que le patient souffre ou non de COVID-19. Les patients qui sont en hémorragie majeure active devraient recevoir les transfusions appropriées, conformément aux protocoles locaux en vigueur.

\section{Références}

1. Tang N, Li D, Wang X, et al. Abnormal coagulation parameters are associated with poor prognosis in patients with novel coronavirus pneumonia. J Thromb Haemost 2020;18:844-7.

2. Zhou F, Yu T, Du R, et al. Clinical course and risk factors for mortality of adult inpatients with COVID-19 in Wuhan, China: a retrospective cohort study. Lancet 2020;395:1054-62.

3. Richardson S, Hirsch JS, Narasimhan M, et al. Presenting characteristics, comorbidities, and outcomes among 5700 patients hospitalized with COVID-19 in the New York City area. JAMA le 22 avril 2020 [Cyberpublication avant impression]. doi: 10.1001/jama.2020.6775.

4. Cui S, Chen S, Li X, et al. Prevalence of venous thromboembolism in patients with severe novel coronavirus pneumonia. $J$ Thromb Haemost le 9 avril 2020 [Cyberpublication avant impression]. doi: 10.1111/jth.14830.

5. Klok FA, Kruip MJHA, van der Meer NJM, et al. Incidence of thrombotic complications in critically ill ICU patients with COVID-19. Thromb Res le 10 avril 2020 [Cyberpublication avant impression]. pii: S0049-3848(20)30120-1. doi: 10.1016/j. thromres.2020.04.013.

6. Tang N, Bai H, Chen X, et al. Anticoagulant treatment is associated with decreased mortality in severe coronavirus disease 2019 patients with coagulopathy. J Thromb Haemost le 27 mars 2020 [Cyberpublication avant impression]. doi: 10.1111/ jth.14817.

Intérêts concurrents : Michael Fralick est coinvestigateur et Michelle Sholzberg est investigatrice principale d'un essai clinique sur des patients présentant une coagulopathie associée à la COVID19. Aucun autre intérêt concurrent n'a été déclaré.

Cet article a été révisé par des pairs.

Affiliations : Hôpital St. Michael (Lee) et Département de médecine, Université de Toronto; Réseau de santé Sinaï et Département de médecine (Fralick), Université de Toronto; Hôpital St. Michael et Département de médecine, et de médecine de laboratoire et pathobiologie (Sholzberg), Institut du savoir Li Ka Shing, Université de Toronto, Toronto, Ont.

Correspondance : Michelle Sholzberg, Michelle. Sholzberg@unityhealth.to 